\title{
NOUVELle
}

\section{Cibler la protéase majeure du SARS-CoV-2 pour fabriquer un médicament efficace contre ce coronavirus}

\author{
Annie Ladoux ${ }^{1}$, Stéphane Azoulay ${ }^{2}$, Christian Dani ${ }^{1}$
}

\begin{abstract}
> Nous sommes de plus en plus souvent confrontés à des maladies infectieuses à l'origine d'épidémies, pour lesquelles il n'existe pas encore de traitement efficace. La plupart de ces épidémies sont dues à l'émergence de microorganismes pathogènes, notamment des virus, qui se transmettent naturellement d'animaux vertébrés aux humains. L'ampleur de l'épidémie va ensuite dépendre de la capacité de transmission du pathogène entre individus contaminés.
\end{abstract}

En décembre 2019, un nouveau coronavirus, SARS-CoV-2 (severe acute respiratory syndrome-coronavirus-2), a causé un syndrome respiratoire aigu sévère (SRAS) chez de nombreux habitants de la province du Hubei en Chine, et, à la faveur des voyages intercontinentaux, s'est rapidement répandu dans le monde entier [1]. Mijuin 2020, on comptait plus de 7,7 millions de cas d'infection confirmée par ce virus (coronavirus disease-19, COVID-19), dont plus de 427000 ayant entrainé le décès (COVID-19 Dashboard by the Center for Systems Sciences and Engineering [CSSE] at Johns Hopkins University), ce qui en fait la pandémie la plus grave de ces dernières décennies. Comme il n'existe à ce jour aucun médicament pour soigner cette infection, des essais cliniques sont en cours pour évaluer l'efficacité de différents médicaments disponibles sur le marché, dont certains sont utilisés pour traiter le Sida (syndrome d'immunodéficience acquise), dû à une infection chronique par le VIH (virus de l'immunodéficience humain). Quels sont ces derniers médicaments et comment agissent-ils pour limiter l'infection?

\section{Les coronavirus et leur propagation}

Les coronavirus provoquent généralement des infections bénignes des voies respiratoires, comme une rhinite. Cependant, en 2003, un coronavirus dénommé SARSCoV-1 a été reconnu responsable d'un SRAS ayant causé la mort de plus de 750 personnes dans le monde [2] $(\rightarrow)$.

$(\rightarrow)$ Voir le Repères d'l. Tratner, $m / s n^{\circ} 8-9$, août-septembre2003, page 885

Les génomes du SARS-CoV-1 et du SARSCoV-2 présentent $79 \%$ d'identité dans leurs séquences nucléotidiques, et les protéines virales synthétisées ont donc, elles aussi,

\author{
'Université Côte d'Azur, Institut de biologie \\ Valrose, Faculté de médecine, \\ 28 avenue Valombrose, 06107 Nice, France. \\ 'Université Côte d'Azur, Institut de chimie de \\ Nice, Faculté des sciences, 28 avenue Valrose, \\ 06108 Nice, France. \\ ladoux@unice.fr
}

des structures très semblables [3]. Le génome des coronavirus est composé d'un simple brin d'acide ribonucléique (ARN) portant l'information pour produire plusieurs protéines qui permettent au virus de se multiplier dans la cellule infectée et de créer de nouvelles particules virales infectieuses. La première protéine produite est une réplicase virale (ARN polymérase ARN-dépendante), une enzyme qui fabrique des nouvelles molécules d'ARN en prenant comme modèle le brin d'ARN viral. Ces ARN nouvellement synthétisés sont ensuite traduits par les ribosomes et enzymes de la cellule infectée pour produire les protéines qui formeront les nouvelles particules virales (virions). Une longue poly-protéine unique est d'abord synthétisée. Elle sera ensuite coupée par l'une des deux protéases virales issues de cette poly-protéine par auto-clivage [18] $(\rightarrow)$ en protéines de tailles plus petites, qui formeront notamment la capside virale, c'està-dire la coque dans

$\rightarrow$ Voir la Synthèse de D. Juckel et al., page 633 de ce numéro laquelle l'ARN viral sera « empaqueté ». C'est ainsi que se forment les nouveaux virus infectieux. 


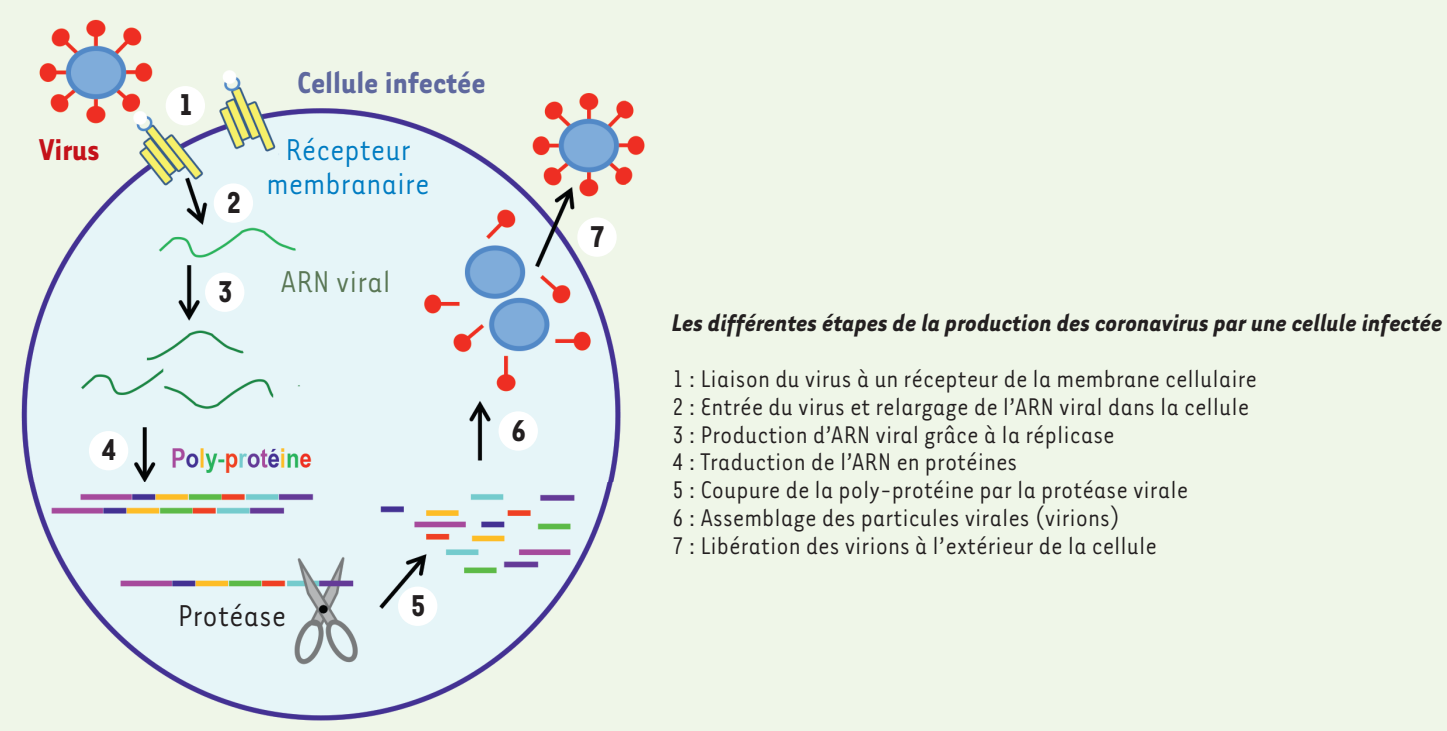

Figure 1. Représentation schématique de la production de particules virales par une cellule.

Plusieurs étapes sont donc nécessaires pour la formation de nouvelles particules virales: entrée du virus dans la cellule qu'il va infecter, copie de l'ARN viral, synthèse de la poly-protéine et sa découpe en protéines fonctionnelles de tailles plus petites, et enfin, reconstitution de nouvelles particules virales infectieuses et leur relargage à l'extérieur de la cellule infectée (Figure l).

\section{Comment peut-on limiter l'infection par les coronavirus?}

Plusieurs stratégies existent pour lutter contre les virus :

1) On peut envisager de bloquer l'entrée du virus dans la cellule si on connaît le «récepteur» de la membrane plasmique sur lequel il se fixe, ce qui n'est pas toujours le cas. Parfois, cette stratégie s'avère impossible à mettre en œuvre car le récepteur en question est nécessaire à d'autres fonctions cellulaires vitales.

2) On peut tenter d'empêcher la formation d'ARN viral.

3) On peut aussi envisager de bloquer la protéase du virus pour l'empêcher de couper la poly-protéine virale synthétisée par la cellule infectée. Les particules virales ne pourront ainsi pas s'assembler dans la cellule, ce qui stoppera l'infection. Cette stratégie a été utilisée avec succès pour traiter plusieurs infections virales dans des maladies comme le Sida ou l'hépatite $C$, bien que cela ne permette pas d'éliminer complètement le virus de l'organisme hôte.

\section{Les protéases virales, des cibles de} choix pour fabriquer des médicaments antiviraux

Les protéases virales sont essentielles à la production de virions par la cellule infectée car elles participent à une étape de maturation en coupant les poly-protéines virales synthétisées à des sites bien précis. Elles sont spécifiques d'un virus donné et agissent sur les protéines virales, ainsi que sur quelques protéines de la cellule hôte, pour favoriser la réplication virale [4]. C'est pour cela qu'elles suscitent un grand intérêt pour stopper la propagation des épidémies virales.

Le premier inhibiteur de la protéase (IP) du VIH, le saquinavir, a été mis sur le marché en 1995 pour traiter les malades atteints du Sida [5]. Depuis, d'autres inhibiteurs de cette protéase ont été développés pour lutter contre la maladie, comme le lopinavir [6] qui, selon l'Organisation mondiale de la santé (OMS), est un des médicaments essentiels pour traiter le Sida. Ces médicaments ciblant la protéase virale, souvent prescrits en association avec ceux qui ciblent la réplicase, ont permis de réduire la charge virale des individus atteints de la maladie.

Cependant les IP dirigés contre la protéase du VIH ont des effets indésirables, dont les mécanismes sont étudiés par notre laboratoire depuis plus de dix ans. Ils induisent notamment une redistribution marquée du tissu adipeux ou lipodystrophie, préjudiciable: le tissu adipeux sous-cutané s'atrophie et la masse grasse viscérale augmente. De plus, les lipodystrophies vont de pair avec des altérations métaboliques comme une hyperlipidémie ou une résistance à l'insuline, qui sont à l'origine de maladies cardio-vasculaires [7, 8] $(\rightarrow)$.

Nous avons mon- $(\rightarrow)$ Voir la Synthèse de J. Capeau et al., $m / s n^{\circ} 5$, mai 2006, page 531 tré que les IP empêchaient la formation d'adipocytes, qu'ils modifiaient les précurseurs adipocytaires ainsi que le métabolisme des adipocytes [9-11], et nous avons caractérisé le mécanisme par lequel ils induisent une résistance à l'insuline [12]. 


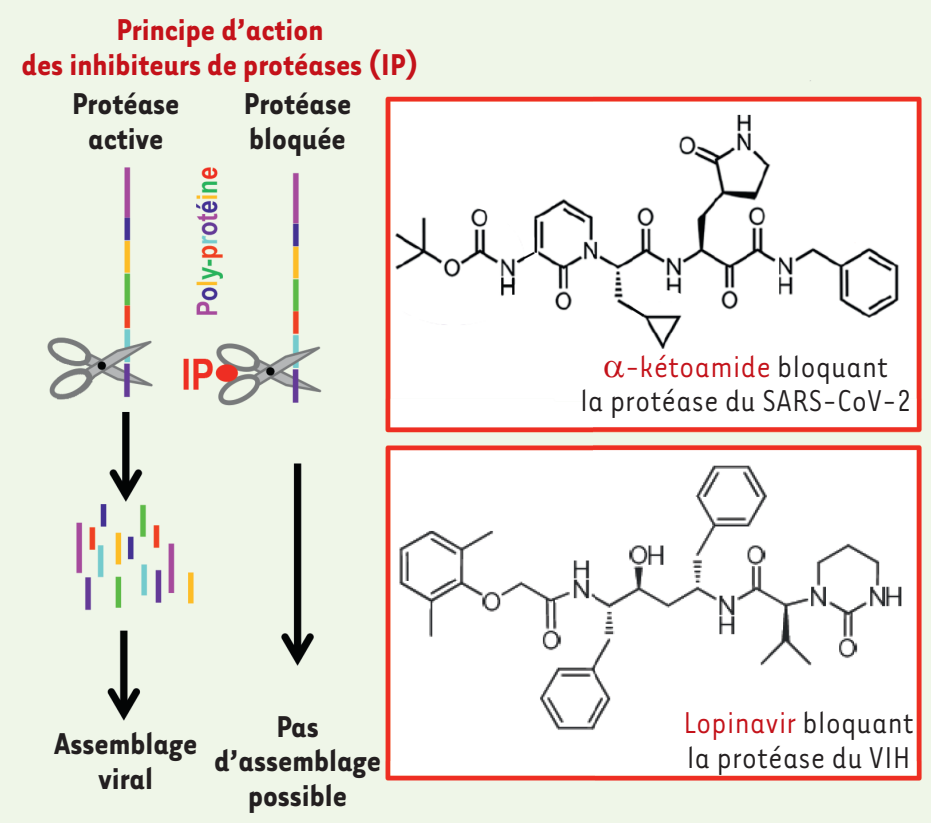

Figure 2. Principe d'action des inhibiteurs des protéases et comparaison des structures chimiques de deux inhibiteurs de protéases virales : le lopinavir et un $\alpha$-kétoamide.

\section{Identification de la protéase majeure du SARS-Cov-2}

Pour comprendre le fonctionnement des protéases virales, il faut certes connaître leur séquence d'acides aminés, mais aussi leur structure tri-dimensionnelle. Cela permet alors de caractériser précisément le site catalytique de l'enzyme, qui sera la cible des molécules à usage thérapeutique capables de le bloquer. Les coronavirus possèdent deux protéases [13]. La structure cristalline de la protéase majeure du SARSCoV-2, 3CL ${ }^{\text {ro }}$ (3C-like proteinase), vient d'être publiée [14]. Sa séquence protéique est identique à $96 \%$ à celle des protéases $3 \mathrm{CL}^{\text {pro }}$ des autres coronavirus, qui ont été très étudiées $[15,18]$

$(\rightarrow)$. Elle coupe la poly-protéine virale 11 fois au niveau de $(\rightarrow)$ Voir la Synthèse de D. Juckel et al., page 633 de ce numéro motifs Leu-Gln-(Ser/Ala/Gly). La deuxième protéase du SARS-CoV-2, PL pro (papain-like protease), a des propriétés différentes de celles de $3 \mathrm{CL}^{\text {pro, }}$, notamment une activité déubiquitinase, et elle pourrait intervenir pour altérer la réponse immunitaire innée de l'hôte infecté. La structure cristalline de cette protéase n'est pas encore connue.
Le principe d'action des molécules bloquant les protéases est schématisé dans la Figure 2. Des inhibiteurs de la protéase $3 C^{\text {pro }}$ des coronavirus déjà connus ont été testés pour leur capacité à inhiber celle du SARSCoV-2. Une molécule de la famille des $\alpha$-kétoamides a été retenue pour son efficacité d'insertion dans le site catalytique de la protéase, ce qui bloque son activité et permet de réduire considérablement la production virale dans des cellules de poumon humain infectées avec le SARS-CoV-2 in vitro, ainsi que dans des modèles d'études précliniques [14]. La tolérance de cette molécule par l'organisme, la voie d'administration la plus adaptée, et ses effets indésirables devront être évalués avant de pouvoir éventuellement l'utiliser pour traiter les patients atteints du COVID-19.

\section{Les médicaments ciblant la protéase} du VIH peuvent-ils bloquer la protéase $3 \mathrm{CL}^{\text {pro }}$ du SARS-CoV-2 ?

Comme il n'y a pas encore de médicament contre le SARS-CoV-2, des essais cliniques pour tester l'efficacité des inhibiteurs de la protéase du VIH sur le COVID-19 ont été menés en Chine [16], et d'autres sont en cours en Europe. Le VIH n'est pas apparenté aux coronavirus, et la réplication de son matériel génétique s'effectue par un mécanisme différent. Mais il produit lui aussi une poly-protéine qui doit être coupée 9 fois par une protéase pour former les nouvelles particules virales. Cette protéase n'appartient pas à la même famille que celle du SARS-CoV-2 puisqu'il s'agit d'une protéase aspartique, comme les pepsines, les cathepsines, et les rénines, qui sont présentes naturellement dans les cellules eucaryotes [17]. Pour la protéase du VIH également, les données de la cristallisation ont grandement facilité la conception d'IP efficaces. Aujourd'hui, il en existe dix approuvés par la Food and drug administration aux États-Unis, dont le mélange lopinavir-ritonavir qui a été testé chez les malades atteints du COVID-19. L'essai clinique chinois a inclus 199 patients, dont 99 ont été traités par les IP du VIH tandis que 100 ont reçu des soins standards. Les résultats indiquent que les IP du VIH n'apportent pas de bénéfice pour traiter les patients atteints de COVID-19. Leurs effets indésirables ont même conduit à arrêter prématurément le traitement chez 13 patients [16]. Ce résultat, bien que décevant, est en accord avec les différences de structure et de fonctionnement entre les protéases du VIH et du SARS-CoV-2. Dans l'essai clinique adaptatif européen Discovery coordonné par l'Inserm et dans l'essai international Solidarity initié par l'OMS, le lopinavir et le ritonavir, associés ou non à l'interféron $\beta$-la, ont été inclus en plus des soins standards, pour évaluer leurs effets contre le SARS-CoV-2. Les résultats devraient apporter un éclairage supplémentaire sur la pertinence d'utilisation des IP du VIH pour lutter contre des infections virales dues à un virus différent.

\section{En conclusion}

La stratégie la plus rapide pour tenter de trouver des médicaments efficaces contre le COVID-19 est de mener des 
essais cliniques avec des médicaments déjà utilisés contre d'autres maladies, puisque l'on sait comment les administrer et qu'on en connaît les doses efficaces (stratégie du «repositionnement de médicament », ou drug repositioning en anglais). Cependant, les inhibiteurs d'une protéase donnée ne sont pas nécessairement efficaces pour bloquer une autre protéase, qui a des propriétés structurales et fonctionnelles différentes. Des molécules de la famille des $\alpha$-kétoamides, capables de bloquer la protéase $3 \mathrm{CL}^{\text {pro }}$ du SARS-CoV-2, représentent un espoir réel pour traiter les patients. Certaines ont déjà été utilisées en clinique, comme par exemple le Telaprevir ou le Boceprevir, qui furent parmi les premiers inhibiteurs de protéase prescrits dès 2011 pour traiter les patients atteints d'hépatite $C$. Le développement d'inhibiteurs de protéase devrait bénéficier de moyens conséquents pour pouvoir disposer rapidement d'un traitement efficace contre le COVID-19. $\diamond$

SARS-CoV-2 protease: an excellent target to develop drugs against COVID-19

\section{LIENS D'INTÉRÊT}

Les auteurs déclarent n'avoir aucun lien d'intérêt concernant les données publiées dans cet article.

\section{RéFÉRENCES}

1. Coronaviridae study group of the international committee on taxonomy of $\mathrm{V}$. The species severe acute respiratory syndrome-related coronavirus: classifying 2019-nCoV and naming it SARS-CoV-2. Nat Microbiol 2020 ; 5 : 536-44.

2. Tratner I. SRAS : 1. Le virus. Med Sci (Paris) $2003 ; 19$ : 885-91.

3. Lee EYP, Ng MY, Khong PL. COVID-19 pneumonia: what has CT taught us? Lancet Infect Dis $2020 ; 20$ : 384-5.

4. Jager $\mathrm{S}$, Cimermancic $P$, Gulbahce $N$, et al. Global landscape of HIV-human protein complexes. Nature $2011 ; 481: 365-70$.

5. James JS. Saquinavir (Invirase): first protease inhibitor approved--reimbursement, information hotline numbers. AIDS Treatment News 1995 ; 1-2.

6. Hurst M, Faulds D. Lopinavir. Drugs $2000 ; 60$ : 1371-9 ; discussion 80-1.

7. Capeau J, Caron M, Vigouroux C, et al. Les lipodystrophies secondaires aux traitements antirétroviraux de l'infection par le VIH. Med Sci (Paris) $2006 ; 22: 531-6$.

8. Carr A, Cooper DA. Adverse effects of antiretroviral therapy. Lancet $2000 ; 356: 1423-30$.

9. Ravaud C, Pare M, Azoulay S, et al. Impairment of the activin A autocrine loop by lopinavir reduces selfrenewal of distinct human adipose progenitors. Sci Rep $2017 ; 7: 2986$.

10. Ravaud C, Paré M, Yao X, et al. Resveratrol and HIVprotease inhibitors control UCPl expression through opposite effects on p38 MAPK phosphorylation in human adipocytes. J Cell Physiol 2019 ; 235 : 1184-96.
11. Vernochet C, Azoulay S, Duval D, et al. Human immunodeficiency virus protease inhibitors accumulate into cultured human adipocytes and alter expression of adipocytokines. J Biol Chem 2005 ; 280 : 2238-43.

12. Djedaini M, Peraldi P, Drici MD, et al. Lopinavir co-induces insulin resistance and $\varepsilon R$ stress in human adipocytes. Biochem Bioph Res Co 2009 ; 386 : 96-100

13. Hilgenfeld R. From SARS to MERS: crystallographic studies on coronaviral proteases enable antiviral drug design. FEBS J 2014 ; 281 : 4085-96.

14. Zhang L, Lin D, Sun X, et al. Crystal structure of SARS-CoV-2 main protease provides a basis for design of improved $\alpha$-ketoamide inhibitors. Science 2020 ; 368 : 409-12.

15. Anand K, Ziebuhr J, Wadhwani P, et al. Coronavirus main proteinase $\left(3 \mathrm{CL}^{\text {pro }}\right)$ structure: basis for design of anti-SARS drugs. Science $2003 ; 300: 1763-7$.

16. Cao B, Wang $Y$, Wen $D$, et al. A trial of lopinavirritonavir in adults hospitalized with severe Covid-19. N Engl J Med 2020 ; 382 : 1787-99.

17. Lapatto R, Blundell T, Hemmings A, et al. X-ray analysis of HIV-1 proteinase at $2.7 \AA$ resolution confirms structural homology among retroviral enzymes. Nature 1989 ; 342 : 299-302.

18. Juckel D, Dubuisson J, Belouzard S. Les coronavirus, ennemis incertains. Med Sci (Paris) 2020, 36: 633-41.
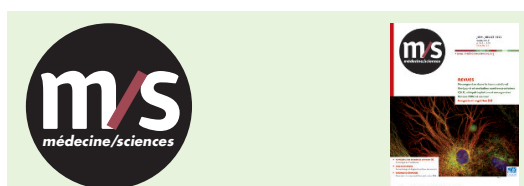

Abonnez-vous

à médecine/sciences

Bulletin d'abonnement page 678 dans ce numéro de $\mathrm{m} / \mathrm{s}$

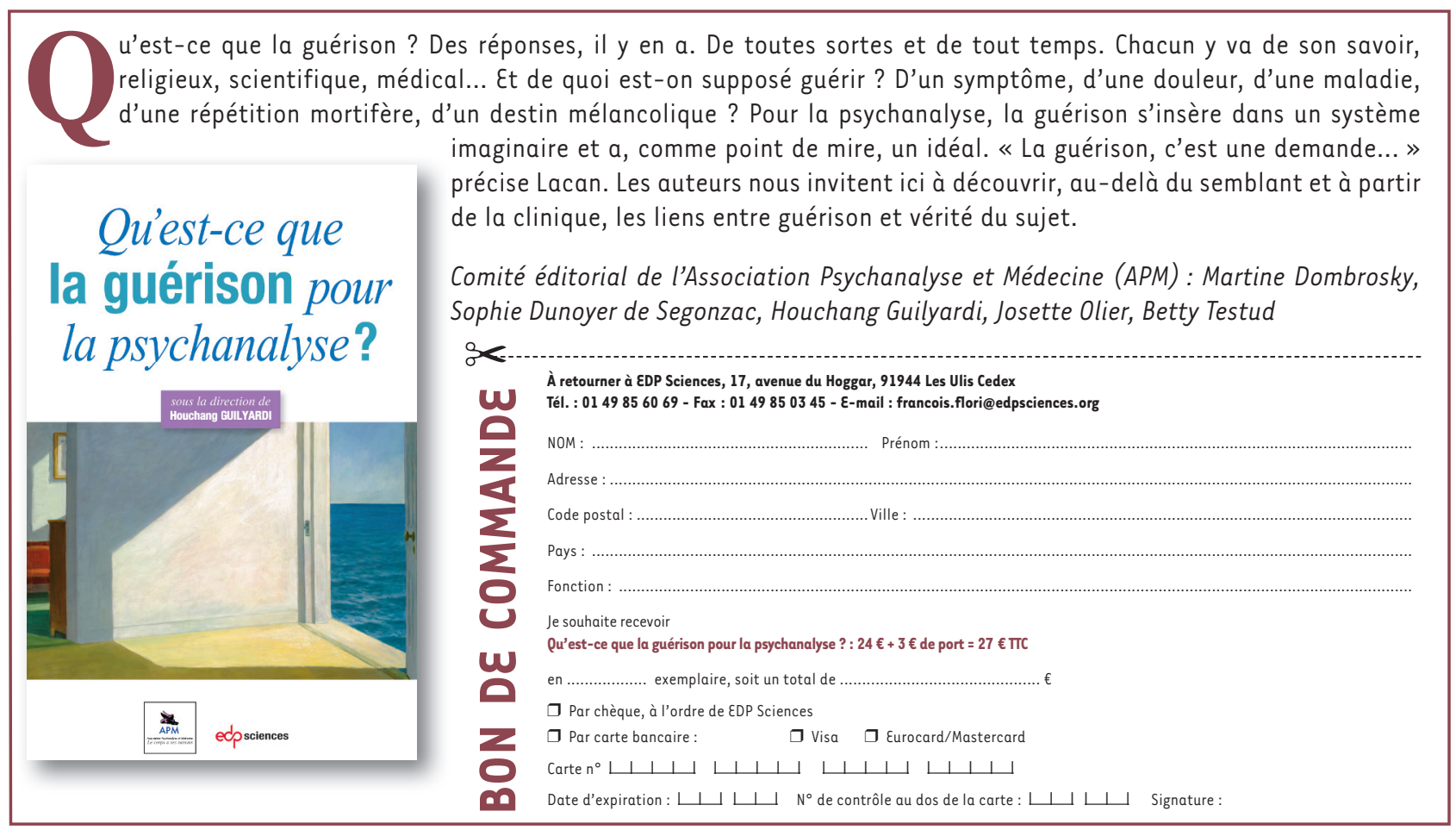

